\title{
Combination of GI-RADS and 3D-CEUS for differential diagnosis of ovarian masses
}

1. Department of Ultrasound, Zhangzhou Affiliated Hospital of Fujian Medical University, Zhangzhou 363000, China 2. Department of Clinical Medicine, Quanzhou Medical College, Quanzhou 362000 , China 3. Department of Ultrasound, the Second Affiliated Hospital of Fujian Medical University, Quanzhou 362000, China

http://dx.doi.org/10.1590/1806-9282.65.7.959

\section{SUMMARY}

OBJECTIVE: The purpose of this study is to evaluate the efficacy of the combination of gynecologic imaging reporting and data system (GI-RADS) ultrasonographic stratification and three-dimensional contrast-enhanced ultrasonography (3D-CEUS) in order to distinguish malignant from benign ovarian masses.

METHODS: In this study, 102 patients with ovarian masses were examined by both two-dimensional ultrasound(2D-US) and 3D-CEUS. Sonographic features of ovarian masses obtained from 3D-CEUS were analyzed and compared with 2D-US. All patients with ovarian masses were confirmed by operational pathology or long-term follow-up results.

RESULTS:(1)The Chi-square test and multiple Logistic regression analysis confirmed that there were only eight independent predictors of malignant masses, including thick septa $(\geq 3 \mathrm{~mm})$, thick papillary projections $(\geq 7 \mathrm{~mm})$, solid areas, presence of ascites, central vascularization, contrast enhancement, distribution of contrast agent, and vascular characteristics of the solid part and their odds ratios which were 5.52, 5.39, 4.94, 4.34, 5.92, 7.44, 6.09, and 7.67, respectively ( $P<0.05)$. (2)These eight signs were used to combine the GI-RADS with 3D-CEUS scoring system in which the corresponding value of the area under the curve (AUC) was 0.969, which was superior to using GI-RADS lonely (Z-value $=1.64, P<0.025)$. Using 4 points as the cut-off, the scoring system showed the performance was clearly better than using GI-RADS alone ( $P<0.05)$. (3) The Kappa value was 0.872 for two different clinicians with equal experience.

CONCLUSIONS: The combination of GI-RADS and 3D-CEUS scoring system would be a more effective method to distinguish malignant from benign ovarian masses.

KEYWORDS: Neoplasias ovarianas. Doenças ovarianas. Ultrasonography. Imaging, three-dimensional/methods.

\section{INTRODUCTION}

Ovarian cancer is the fifth most common type of cancer and the most aggressive. Despite advances in surgery, chemotherapy, and intensive ongoing researches, 5-year survival has not significantly increased. ${ }^{1}$ Thus, the early detection of ovarian tumors and accurate assessment of their properties are still the major issues attracting attention in the medical community. ${ }^{2}$ Currently, ultrasonic determination of

DATE OF SUBMISSION: 02-May-2018

DATE OF ACCEPTANCE: 05-Aug-2018

CORRESPONDING AUTHOR:Guorong LV

Zhangzhou - Zhangzhou - 363000

Tel: +86-18150605088

E-mail: guoronglv@outlook.com 
benign or malignant ovarian tumors primarily involves subjective and qualitative diagnosis. There is a lack of quantitative information and a strong dependence on the physicians' experience and operative techniques. ${ }^{3}$ Therefore, semi-quantitative sonographic scoring system (SSS) has been proposed, with sensitivity, specificity, and diagnostic accuracy significantly higher than using a conventional two-dimensional ultrasound (2DUS) method. ${ }^{4}$ The SSS can be utilized to score a tumor based on its 2D-US sonographic characteristics, including size, shape, border, wall thickness, internal echo characteristics, septa, posterior shadowing, presence of ascites, color flow distribution, and other factors. In addition, SSS would be appropriate to incorporate a multi-mode diagnostic ultrasound system including power Doppler sonography, three-dimensional power Doppler (3D-PD) sonography, contrast-enhanced ultrasonography (CEUS), and 3D contrast-enhanced power Doppler ultrasonography (3D-CE-PDU) into the scoring system. ${ }^{5-7}$ However, the sonographic characteristics of the selected SSS were baseless and unjustified.

In 2009, Amor et al. ${ }^{8}$ proposed a gynecologic imaging reporting and data system (GI-RADS) for reporting results in adnexal masses based on six characteristics including thick septa ( $\geq 3 \mathrm{~mm})$, thick papillary projections $(\geq 7 \mathrm{~mm})$, solid areas, presence of ascites, central vascularization and resistance index $(\mathrm{RI})<0.5$, sensitivity, specificity, positive predictive value (PPV), and negative predictive value (NPV), which were $92 \%, 97 \%$, $85 \%$, and $99 \%$, respectively. A new transvaginal ultrasonographic technique called 3D contrast-enhanced ultrasonography (3D-CEUS) added more information by incorporating 3D imaging with CEUS and has been confirmed as superior to 2Dcontrast-enhanced ultrasonography (2D-CEUS) in differentiating benign and malignant ovarian tumors. ${ }^{9}$ In this study, we describe and propose a new scoring system including six factors from GI-RADS and four factors from 3D-CEUS, which is, in fact, a combination of GI-RADS and the 3D-CEUS scoring system for conducting a semi-quantitative evaluation of the nature of ovarian tumors as well as comparing the diagnostic accuracy of this new SSS method with that of GI-RADS.

\section{METHODS}

In this study, 102 women (age range 16-68 years; mean age $32.8 \pm 12.2$ years) with diagnoses of ovarian masses participated with cystic-solid, solid, thick separation, or thick papillary projections on B-mode between July 2015 and July 2016. Exclusion criteria were pregnancy, breastfeeding, severe heart failure based on the New York Heart Association (NYHA) classes (class IV was adopted), and ultrasound contrast agents (UCAs) contraindicated in patients. The patients or their legally authorized representatives consented to the treatment by signing and dating the informed consent documents. This study was approved by the institutional review board (IRB) and followed the Helsinki Declaration for human studies. ${ }^{10}$

\section{D-US}

All 2D-US examinations were performed using the Voluson E8 Expert (GE Medical Systems, MA, USA) ultrasound machine with dedicated 3D imaging software. The vaginal probe model was RIC5-9-D, with a frequency range of 5.0-9.0 MHz, and a mechanical index (MI) of 0.08. The 2D-US examinations were undertaken at our department by experienced sonographers (>10,000 pelvic sonographies). After the examinations, the GI-RADS was used with the following classifications. GI-RADS 1, definitively benign; normal ovaries were identified, and no adnexal mass was seen. GI-RADS 2, very probably benign; this category included adnexal lesions thought to be of functional origin, such as follicles, corpora lutea, and hemorrhagic cysts. GI-RADS 3, probably benign; this category included neoplastic adnexal lesions thought to be benign, such as endometrioma, teratoma, simple cyst, hydrosalpinx, paraovarian cyst, peritoneal pseudocyst, pedunculated myoma, and findings suggestive of pelvic inflammatory disease. GI-RADS 4, probably malignant; this category included adnexal lesions that could not be included in the other groups, with 1 or 2 findings suggestive of malignancy (i.e., thick papillary projections, thick septations, solid areas, central vascularization, ascites, and a lowest $\mathrm{RI}<0.5$ ). GI-RADS 5 , very probably malignant; this category included adnexal masses with 3 or more of the findings suggestive of malignancy listed for GI-RADS $4 .^{8}$

\section{D-CEUS}

In this study, a SonoVue contrast agent (Bracco, Milan, Italy) was used. A bolus of $2.4 \mathrm{~mL}$ was injected into the median cubital vein and was immediately followed by injections of 5-10 mL saline. As a contrast agent, $59 \mathrm{mg}$ SonoVue was added to $5 \mathrm{~mL}$ saline and mixed well. The scanning was carried out by steadily 
moving the probe from top to bottom using freehand static 3D-CEUS mode, and the sampling was repeated every 10 seconds until 90 seconds. All 3D-CEUS images were stored.

The analysis was made through rotation and tomographic ultrasound imaging (TUI). The four following criteria were observed: enhanced time, contrast enhancement, distribution of contrast agent in enhanced solid part, vascular characteristics of solid part, quickly enhanced time, significant enhancement, inhomogeneous distribution of contrast agent in enhanced solid part, and abnormally vascular characteristics of solid part were detected as malignancy signs.

The reference standard for the final diagnosis of a benign or malignant tumor was the histological examination of the operative specimen or long-term follow-up findings.

\section{STATISTICAL ANALYSIS}

In order to do the statistical analysis, data were analyzed using SPSS software(Version 22.0) for Windows (SPSS, Chicago, IL, USA). The Chi-square test and multiple Logistic regression analysis were performed to investigate whether the independent risk predictors in the differential diagnosis of benign and malignant ovarian could be confirmed. In addition, using a combination of GI-RADS and 3D-CEUS scoring system, the receiver operating characteristic (ROC) curve was drawn to determine the cut-off value. The cut-off value was then applied as an original score to calculate sensitivity, specificity, diagnostic accuracy, PPV, and NPV. A Two-tailed $P<0.05$ was considered statistically significant.

Two blinded examiners evaluated 20 consecutive cases. Interobserver reproducibility was assessed using the Kappa index.

\section{RESULTS}

\section{Histological follow-up results}

Among 102 ovarian masses, 67 were benign, which was confirmed by surgical pathology (63 masses) or long-term follow-up (4 masses); the remaining 35 were malignant masses, all pathologically confirmed.

\section{Ten characteristics of ovarian masses}

The Chi-square test showed ten characteristics that can be used to differentiate benign and malignant ovarian masses. However, multiple Logistic regression analysis confirmed that there were only eight independent predictors of malignant masses, including thick papillary projections, thick septa, central vascularization, ascites, solid lesions, contrast-enhanced, distribution of contrast agent, and vascular characteristics of solid tumors $(P<0.05=$ as displayed in Figure1).

Combination of GI-RADS with 3D-CEUS scoring system and comparison of the diagnostic efficiency with GI-RADS

The combination of GI-RADS with 3D-CEUS scoring system based on the mentioned eight independent
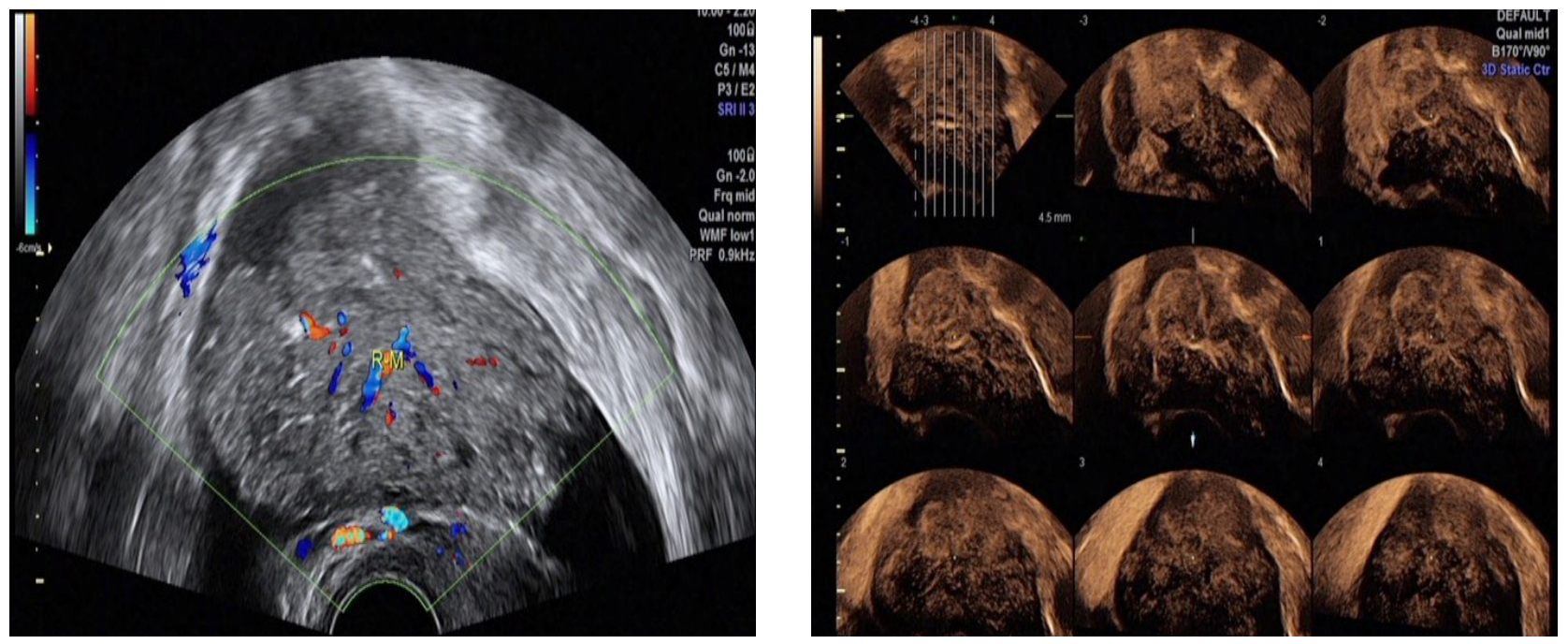

FIGURE 1. A serious adenocarcinoma (stage la). A) Transvaginal sonogram showing a solid area with irregular contours and blood flow within it and ascites (thick arrow). B) 3D-CEUS shows a significant, inhomogeneous enhanced region in the parenchyma. Stereoscopic vascularity and clear courses of blood vessels (thin arrow) can be seen in every layer of the 3D TUI-CEUS. The GI-RADS combination with 3D-CEUS system score was 6. 


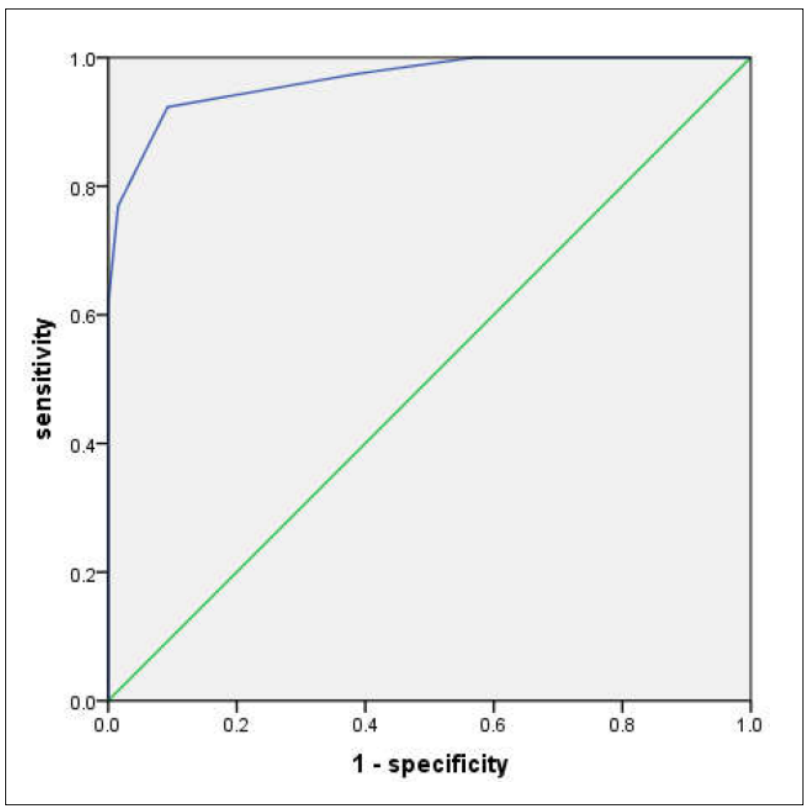

FIGURE 2. ROC curves of the combination of GI-RADS with 3D-CEUS scoring system in distinguishing benign from malignant small adnexal masses.

predictors and the area under the curve (AUC) was 0.969, as depicted in Figure 2, which was superior to the 0.837 of GI-RADS ( $\mathrm{Z}$-value $=1.64, P<0.05=$, as illustrated in Figure 3). It is noteworthy that a score of 4 was chosen as the cut-off, and the sensitivity, specificity, diagnostic accuracy, PPV, and NPV of the new scoring system are all superior to those of
GI-RADS. Furthermore, the same Kappa value of 0.872 was reached by between two different clinicians with equal experience.

\section{DISCUSSION}

Currently, the transvaginal scan (TVS) is the most commonly used and effective approach for detecting and diagnosing ovarian tumors. However, the subjective nature of ultrasound interpretation makes its clinical application somewhat unreliable." In 2012, a scoring system based on surface, wall thickness, inner wall structures, septa, contrast enhancement of the masses, relationship with surrounding tissues and ascites called the 3D-CEUS scoring system was developed by Xiang et al. ${ }^{12}$ with high sensitivity and specificity, $100 \%$ and $98 \%$, respectively. Nevertheless, the selection of factors lacked statistical basis and may be appropriate only for those with a largest diameter smaller than $4 \mathrm{~cm}$. In this study, we attempted to use Chi-square test as primary screening and then multiple Logistic regression analysis to exclude the factors containing collinearity and eventually verified its sensitivity, specificity, and accuracy values for detecting ovarian malignant masses, which were $94.2 \%, 95.5 \%$, and $95.0 \%$, respectively. Furthermore, by covering the signs of GI-RADS containing ovarian mass when the lesions are $>5 \mathrm{~cm}$, an applicable field of combination of GI-RADS and 3D-CEUS scoring system was herein expanded.

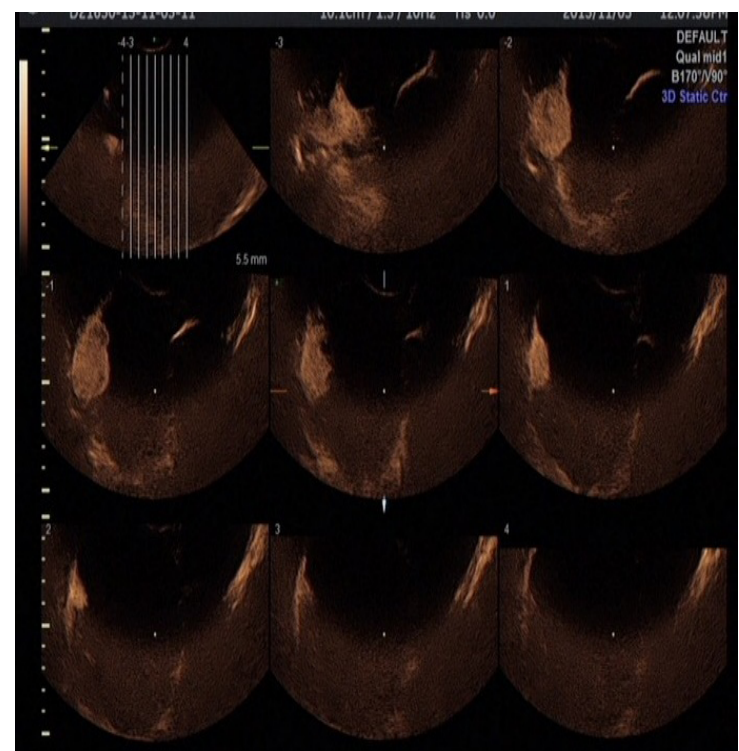

FIGURE3.Comparative analysis of an ovarian mucinous cystadenoma with focal carcinogenesis using TVS (A) and 3D-CEUS(B). In this case, TVS only shows that the solid area is more than 50\% without blood flow within or around it, 3D-CEUS presents more information by clearly showing a significant enhanced, inhomogeneously solid area with disorganized vasculatures that arise from the surface of the internal walls (thick arrow); the GI-RADS combination with 3D-CEUS system score was 4. 
Since its introduction in clinical settings, GI-RADS has been tested extensively in several multicenter studies and shown to be a proper criterion for discriminating between benign and malignant adnexal masses. However, a pilot study reported that a significant number of cystic-solid or solid ovarian masses were confirmed as benign, whereas GI-RADS regarded them as malignant. Thus, the specificity of GI-RADS is still limited..$^{13}$ In this research, we sought to demonstrate that combining GI-RADS and 3D-CEUS scoring system could significantly improve the sensitivity, specificity, and accuracy, in comparison with the use of GI-RADS alone.

Previous studies have shown that the use of 3D-US after the administration of microbubble-based contrast agent, called 3D-CEUS, aids in the characterization of ovarian tumors by evaluating their vascular patterns. ${ }^{14,15}$ The microvascular distribution of ovarian tumors tend to be distorted, complex, and concentrated in different planes, and 3D-CEUS allows the division of structures into tomographic slices in three orthogonal planes overcoming the shortcoming of one single area of interest with 2D-CEUS, thus providing supplementary information which is unachievable by 2D-CEUS. In addition, a recent study demonstrated that 2D-CEUS has no advantages in ovarian masses containing thick papillary projections, which had been recognized as a malignant sign in comparison with conventional US $(P>0.05) .{ }^{16}$ By subsequently presenting several slices through TUI and changing the thickness and distances between two slices, 3D-CEUS can make it easier to detect thick papillary projections which US or 2D-CEUS may miss, it also displays the perfusion condition in its different portions. ${ }^{17}$ It is of note that the peripheral tissue of ovarian tumors can also be displayed sensitively by contrast media, decreasing the resolution of the morphology of ovarian masses by means of 3D-CEUS, while the morphology of ovarian tumors has a significant effect on determining the tumor's natures. ${ }^{18}$ Therefore, combining GI-RADS with 3D-CEUS may help make a comprehensive analysis of the morphologic variations of masses.

To confirm the effectiveness of this advanced scoring system, the number of investigated patients must be increased. In addition, for this purpose, conducting a multicenter study could be efficient. Furthermore, 3D-CEUS cannot show real-time hemodynamic variations in the lesions. This is the reason why we have relied on a sequence of image acquisition to achieve the timing and characteristics of contrast enhancement by comparing with that of the uterus. This limitation similarly affects acquisition on both lesion and uterus. Further studies are required to address these specific issues.

\section{RESUMO}

OBJETIVO: O objetivo deste estudo é avaliar a eficácia da combinação da estratificação por ultrassonografia usando o Sistema de Relatórios e Dados de Imagem Ginecológica (GI-RADS) e ultrassonografia 3D com contraste (3D-CEUS) para diferenciar massas ovarianas benignas de malignas.

METODOLOGIA: Neste estudo, 102 pacientes com massas ovarianas foram examinadas usando ultrassonografia bidimensional (2DUS) e 3D-CEUS. As características ultrassonográficas das massas ovarianas obtidas com 3D-CEUS foram analisadas e comparadas com de 2D-US. Todos os pacientes com massas ovarianas tiveram o diagnóstico confirmado pelos resultados de patologia cirúrgica ou acompanhamento de longo prazo.

RESULTADOS: (1) O teste qui-quadrado e a regressão logística múltipla confirmaram a existência de apenas oito preditores independentes de massas malignas, incluindo septos espessos $(\geq 3 \mathrm{~mm})$, projeções papilares espessas $(\geq 7 \mathrm{~mm})$, áreas sólidas, presença de ascite, vascularização central, aumento de contraste, distribuição do agente de contraste e características vasculares da parte sólida e suas razões de possibilidades (OR), que foram 5,52, 5,39, 4,94, 4,34, 5,92, 7,44, 6,09 e 7.67, respectivamente $(P<0,05)$. (2) Esses oito preditores foram utilizados para combinar o GI-RADS com o sistema de escores da 3D-CEUS, para o qual o valor correspondente da área sob a curva (AUC) foi de 0,969, superior ao uso exclusivo do GI-RADS (valor de $Z=1,64, P<0,025$ ). Usando 4 pontos como corte, o sistema de escores mostrou que o desempenho foi muito melhor do que com o uso exclusivo do GI-RADS $(P<0,05)$. (3) O valor de Kappa foi 0,872, obtido por dois médicos diferentes com igual experiência.

CONCLUSÃO: A combinação do GI-RADS e do sistema de pontuação da 3D-CEUS é um método mais eficaz para distinguir massas ovarianas benignas de malignas.

PALAVRAS-CHAVE: Ovarian neoplasms. Ovarian diseases. Ultrassonografia. Imagem tridimensional/métodos. 


\section{REFERENCES}

1. Siegel RL, Miller KD, Jemal A. Cancer Statistics, 2017. CA Cancer J Clin. 2017;67(1):7-30.

2. Lutz AM, Willmann JK, Drescher CW, Ray P, Cochran FV, Urban N, et al. Early diagnosis of ovarian carcinoma: is a solution in sight? Radiology. 2011;259(2):329-45.

3. Menon U, Gentry-Maharaj A, Hallett R, Ryan A, Burnell M, Sharma A, et al. Sensitivity and specificity of multimodal and ultrasound screening for ovarian cancer, and stage distribution of detected cancers: results of the prevalence screen of the UK Collaborative Trial of Ovarian Cancer Screening (UKCTOCS). Lancet Oncol. 2009;10(4):327-40.

4. Alcázar JL, Mercé LT, Laparte C, Jurado M, López-García G. A new scoring system to differentiate benign from malignant adnexal masses. Am | Obstet Gynecol. 2003;188(3):685-92.

5. Sun LJ, Wu QQ, Chen Y. A study of an ultrasound-based scoring system for preoperative assessment of ovarian tumors and assisting triage for surgical management. Chin J Med Ultrasound. 2010;7:29-32.

6. Alcázar JL, Mercé LT, García Manero M. Three-dimensional power Doppler vascular sampling: a new method for predicting ovarian cancer in vascularized complex adnexal masses. J Ultrasound Med. 2005;24(5):689-96.

7. liang ZH, Li KT, Tian JW, Ren M. An overview of the development and application of the sonographic scoring system: differentiation of malignant from benign ovarian tumors. Arch Gynecol Obstet. 2016;293(2):303-10.

8. Amor F, Vaccaro H, Alcázar JL, León M, Craig JM, Martinez J. Gynecologic imaging reporting and data system: a new proposal for classifying adnexal masses on the basis of sonographic findings. J Ultrasound Med. 2009;28(3):285-91.

9. Hu R, Xiang H, Mu Y, Feng Y, Gu L, Liu H. Combination of 2- and 3-dimensional contrast-enhanced transvaginal sonography for diagnosis of small adnexal masses. J Ultrasound Med. 2014;33(11):1889-99.
10. World Medical Association Declaration of Helsinki. Ethical principles for medical research involving human subjects. [Accessed 2014 Oct 10]. Available from: http:// www.wma.net/en/30publications/10policies/b3/

11. Buhling KJ, Lezon S, Eulenburg C, Schmalfeldt B. The role of transvaginal ultrasonography for detecting ovarian cancer in an asymptomatic screening population: a systematic review. Arch Gynecol Obstet. 2017;295(5):1259-68.

12. Xiang $H$, Huang $R$, Cheng J, Gulinaer $S$, Hu R, Feng $Y$, et al. Value of three-dimensional contrast-enhanced ultrasound in the diagnosis of small adnexal masses. Ultrasound Med Biol. 2013;39(5):761-8.

13. Alcázar JL, Rodriguez D. Three-dimensional power Doppler vascular sonographic sampling for predicting ovarian cancer in cystic-solid and solid vascularized masses. J Ultrasound Med. 2009;28(3):275-81.

14. Huchon C, Metzger U, Bats AS, Bensaid C, Chatellier G, Azizi M, et al. Value of three-dimensional contrast-enhanced power Doppler ultrasound for characterizing adnexal masses. J Obstet Gynaecol Res. 2012;38(5):832-40.

15. Qiao JJ, Yu J, Yu Z, Li N, Song C, Li M. Contrast-enhanced ultrasonography in differential diagnosis of benign and malignant ovarian tumors. PLoS One. 2015;10(3):e0118872.

16. Testa AC, Timmerman D, Exacoustos C, Fruscella E, Van Holsbeke C, Bokor $D$, et al. The role of CnTI-SonoVue in the diagnosis of ovarian masses with papillary projections: a preliminary study. Ultrasound Obstet Gynecol. 2007;29(5):512-6

17. Ma X, Zhao Y, Zhang B, Ling W, Zhuo H, jia H, et al. Contrast-enhanced ultrasound for differential diagnosis of malignant and benign ovarian tumors: systematic review and meta-analysis. Ultrasound Obstet Gynecol. 2015,46(3):277-83.

18. Liu Z, Yang F, Zhang Y, Yu H, Zhu H, Yang R, et al. Conventional, Doppler and contrast-enhanced ultrasonography in differential diagnosis of ovarian masses. Cell Physiol Biochem. 2016,39(6):2398-408. 